\title{
The Inverse Eigenproblem for Complementary Submatrix of Generalized Jacobi Matrices
}

\author{
Zhi-bin LI and Yun-fei WANG* \\ School of Mathematics and Physics, Dalian Jiaotong University, \\ Dalian, China \\ E-mail:lizhibinky@163.com, wangyunfeiky@163.com
}

\begin{abstract}
This paper deals with the reconstruction of generalized Jacobi matrices from eigen-pairs of the matrix and its complementary submatrix of give up $k$ row and $k$ column. The paper discussed the existence and uniqueness of the question's solution. The possibilities are exanimated by numerical example.
\end{abstract}

Keywords-Generalized Jacobi Matrices; Complementary Submatrix ;Inverse Eigenvalue Problem

\section{BRING FORWARD THE QUESTION}

The Inverse eigenvalue problem for matrices in the problems involved in the field of mathematical physics, geophysics, quantum chemistry, optics, mechanics, structural design, pattern recognition, automatic control, and so on. Many experts and scholars have addressed this problem more extensively and in-depth studied, and get a lot of results. Some have been used in engineering or scientific calculations of practical solutions.

This paper studies the generalized Jacobi matrix with function relationship:

$$
J=\left(\begin{array}{cccccc}
a_{1} & b_{1} & & & & \\
c_{1} & a_{2} & b_{2} & & & \\
& \ddots & \ddots & \ddots & \\
& & & c_{n-2} & a_{n-1} & b_{n-1} \\
& & & & c_{n-1} & a_{n}
\end{array}\right)
$$

In it, $a_{i}, b_{i}, c_{i} \in R, c_{i}=f_{i}\left(b_{i}\right)(i=1,2, \cdots, k-1)$,

$b_{i}=\varphi_{i}\left(c_{i}\right)(i=k, k+1, \cdots, n-2)$

$f_{i}(x)(x \in R), \varphi_{i}(x)(x \in R)$ are real function.

Use $J_{\backslash k}$ to express the n-1-steps matrix which is removed the $k^{\text {th }}$ row and the $k^{\text {th }}$ column. Record

$$
A=\left(\begin{array}{cccccc}
a_{1} & b_{1} & & & & \\
c_{1} & a_{2} & b_{2} & & & \\
& \ddots & \ddots & \ddots & \\
& & & c_{k-3} & a_{k-2} & b_{k-2} \\
& & & & c_{k-2} & a_{k-1}
\end{array}\right)
$$

$$
B=\left(\begin{array}{cccccc}
a_{k+1} & b_{k+1} & & & & \\
c_{k+1} & a_{k+2} & b_{k+2} & & & \\
& \ddots & \ddots & \ddots & \\
& & & c_{n-2} & a_{n-1} & b_{n-1} \\
& & & & c_{n-1} & a_{n}
\end{array}\right)
$$

So,

$$
J_{\backslash k}=\left(\begin{array}{cc}
A & o \\
O & B
\end{array}\right)
$$

Let $\lambda$ be a characteristic value of $n$ order matrix $J$, $\mathrm{x}$ be corresponding characteristics vector, $(\lambda, x)$ is named the characteristic pair of $J$.

This paper presents the following inverse eigenvalue problem for matrices:

Question A : Given the integer $k, 1 \leq k \leq n$, and two vary real number $\lambda, \mu$ and non-zero vector

$$
x=\left(x_{1}, x_{2}, \cdots, x_{n}\right)^{\mathrm{T}} \in R^{n}, y=\left(y_{1}, y_{2}, \cdots, y_{n-1}\right)^{\mathrm{T}} \in R^{n-1}, \mathrm{~S}
$$

truct $J$ to make $(\lambda, x),(\mu, y)$ are the characteristic pairs of $J$ and $J_{\backslash k}$ respectively.

Record

$$
\bar{y}_{i}=y_{i}(i=1,2, \cdots ; k-1), \bar{y}_{k}=0, \bar{y}_{i}=y_{i-1}(i=k+1, k+2, \cdots ; n)
$$


Let

$$
\begin{aligned}
x_{0} & =\bar{y}_{0}=x_{n+1}=\bar{y}_{n+1}=c_{0}=b_{n}=0 \\
D_{i} & =\left|\begin{array}{ll}
x_{i} & x_{i+1} \\
y_{i} & \bar{y}_{i+1}
\end{array}\right|(i=1,2, \cdots, n-1)
\end{aligned}
$$

\section{II.THE SOLUTION OF PROBLEM}

For question A, there are $J x=\lambda x, J_{\backslash k} y=\mu y$, then When $1 \leq i \leq k-1$,

$$
\left\{\begin{array}{l}
a_{i} x_{i}+b_{i} x_{i+1}=\lambda x_{i}-c_{i-1} x_{i-1} \\
a_{i} \bar{y}_{i}+b_{i} \bar{y}_{i+1}=\mu \bar{y}_{i}-c_{i-1} \bar{y}_{i-1}
\end{array}\right.
$$

When $i=k$,

$$
c_{k-1} x_{k-1}+a_{k} x_{k}+b_{k} x_{k+1}=\lambda x_{k}
$$

When $k+1 \leq i \leq n$,

$\left\{\begin{array}{l}c_{i-1} x_{i-1}+a_{i} x_{i}=\lambda x_{i}-b_{i} x_{i+1} \\ c_{i-1} \bar{y}_{i-1}+a_{i} \bar{y}_{i}=\mu \bar{y}_{i}-b_{i} \bar{y}_{i+1}\end{array}\right.$ get

By formulas (8) and (9) elimination $a_{i}$, then we can

$$
b_{i} D_{i}=(\mu-\lambda) x_{i} \bar{y}_{i}+c_{i-1} D_{i-1}(i=1,2, \cdots, k-1)
$$

For (13), if $\quad D_{i} \neq 0(i=1,2, \cdots, k-1) \quad$, so $x_{i}, \bar{y}_{i}(i=1,2, \cdots, k)$ cannot zero at the same time, so $b_{i}=\frac{(\mu-\lambda) x_{i} \bar{y}_{i}+c_{i-1} D_{i-1}}{D_{i}}, c_{i}=f_{i}\left(b_{i}\right)(i=1,2, \cdots, k-1)$

If $x_{i} \neq 0$, from formulas (8), we can get

$$
a_{i}=\frac{\lambda x_{i}-b_{i} x_{i+1}-c_{i-1} x_{i-1}}{x_{i}}(i=1,2, \cdots, k-1)
$$

If $y_{i} \neq 0$, from formulas (9), we can get

$a_{i}=\frac{\mu \bar{y}_{i}-b_{i} \bar{y}_{i+1}-c_{i-1} \bar{y}_{i-1}}{\bar{y}_{i}}(i=1,2, \cdots, k-1)$

From formulas (11) and (12), we can get

$$
c_{i-1} D_{i-1}=b_{i} D_{i}-(\mu-\lambda) x_{i} \bar{y}_{i}(i=n, n-1, \cdots, k+1)
$$

For formulas (14)

,if $D_{i} \neq 0(i=k, k+1, \cdots, n-1)$,

So $x_{i}, y_{i}(i=k, k+1, \cdots, n)$ cannot zero at the same time, so

$$
\begin{gathered}
c_{i-1}=\frac{b_{i} D_{i}-(\mu-\lambda) x_{i} \bar{y}_{i}}{D_{i-1}}(i=n, n-1, \cdots, k+1) \\
b_{i}=\varphi_{i}\left(c_{i}\right)(i=n-1, n-2, \cdots, k)
\end{gathered}
$$

If $x_{i} \neq 0$, from formulas (10) and(11), we can get

$$
a_{i}=\frac{\lambda x_{i}-c_{i-1} x_{i-1}-b_{i} x_{i+1}}{x_{i}}(i=k, k+1, \cdots, n)
$$

If $y_{i} \neq 0$, from formulas (12), we can get

$$
a_{i}=\frac{\mu \bar{y}_{i}-c_{i-1} \bar{y}_{i-1}-b_{i} \bar{y}_{i+1}}{\bar{y}_{i}}(k+1, k+2, \cdots, n)
$$

Above all,for the question A,we can get

Theorem 1 If $D_{i} \neq 0(i=1,2, \cdots, n-1)$,so the question $\mathrm{A}$ has the only solution,and

$$
\begin{gathered}
b_{i}=\frac{(\mu-\lambda) x_{i} \bar{y}_{i}+c_{i-1} D_{i-1}}{D_{i}}, c_{i}=f_{i}\left(b_{i}\right)(i=1,2, \cdots, k-1) \\
c_{i-1}=\frac{b_{i} D_{i}-(\mu-\lambda) x_{i} \bar{y}_{i}}{D_{i-1}}(i=n, n-1, \cdots, k+1),
\end{gathered}
$$

$$
b_{i}=\varphi_{i}\left(c_{i}\right)(i=n-1, n-2, \cdots, k)
$$

$$
a_{i}=\left\{\begin{array}{l}
\frac{\lambda x_{i}-c_{i-1} x_{i-1}-b_{i} x_{i+1}}{x_{i}}, x_{i} \neq 0 ; \\
\frac{\mu \bar{y}_{i}-c_{i-1} \bar{y}_{i-1}-b_{i} \bar{y}_{i+1}}{\bar{y}_{i}}, \bar{y}_{i} \neq 0
\end{array}(i=1,2, \cdots, n)\right.
$$

III. NUMERICAL EXAMPLE

Example For question A, let $\lambda=-1, \mu=1, x=(1,6,-1,1,2)^{\mathrm{T}}, y=(0,1,2,-1)^{\mathrm{T}}, k=3$.

$$
f_{1}(x)=x+1, f_{2}(x)=|x|-1, \varphi_{3}(x)=5 x, \varphi_{4}(x)=x^{2}
$$

It

is

easy

to 
calculate $\bar{y}=(0,1,0,2,-1), D_{1}=1, D_{2}=1, D_{3}=-2, D_{4}=-5$ satisfied the conditions of theorem 1 , so question $\mathrm{A}$ has the only solution.

$$
\begin{gathered}
b_{1}=\frac{(\mu-\lambda) x_{1} \bar{y}_{1}}{D_{1}}=0, c_{1}=b_{1}+1=1 ; \\
b_{2}=\frac{(\mu-\lambda) x_{2} \bar{y}_{2}+c_{1} D_{1}}{D_{2}}=13, c_{2}=\left|b_{2}\right|-1=12 ; \\
c_{4}=\frac{-(\mu-\lambda) x_{5} \bar{y}_{5}}{D_{4}}=\frac{-4}{5}, b_{4}=c_{4}^{2}=\frac{16}{25} ; \\
c_{3}=\frac{b_{4} D_{4}-(\mu-\lambda) x_{4} \bar{y}_{4}}{D_{3}}=\frac{18}{5}, b_{3}=5 c_{3}=18 ; \\
a_{1}=\frac{\lambda x_{1}-b_{1} x_{2}}{x_{1}}=-1 ; \\
a_{4}=\frac{\lambda x_{4}-c_{3} x_{3}-b_{4} x_{5}}{x_{4}}=\frac{33}{25} ; \\
a_{2}=\frac{\mu \bar{y}_{2}-c_{1} \bar{y}_{1}-b_{2} \bar{y}_{3}}{\bar{y}_{2}}=1 ; \\
a_{3}=\frac{\lambda x_{3}-c_{2} x_{2}-b_{3} x_{4}}{x_{3}}=89 ;
\end{gathered}
$$

So

$$
J=\left(\begin{array}{ccccc}
-1 & 0 & 0 & 0 & 0 \\
1 & 1 & 13 & 0 & 0 \\
0 & 12 & 89 & 18 & 0 \\
0 & 0 & \frac{18}{5} & \frac{33}{25} & \frac{16}{25} \\
0 & 0 & 0 & \frac{-4}{5} & \frac{-3}{5}
\end{array}\right)
$$

$$
J_{\backslash 3}=\left(\begin{array}{cccc}
-1 & 0 & 0 & 0 \\
1 & 1 & 0 & 0 \\
0 & 0 & \frac{33}{25} & \frac{16}{25} \\
0 & 0 & \frac{-4}{5} & \frac{-3}{5}
\end{array}\right)
$$

And $\lambda=-1, x=(1,6,-1,1,2)^{\mathrm{T}}$ is the characteristic pair of $J, \mu=1, y=(0,1,2,-1)^{\mathrm{T}}$ is the characteristic pair of $J_{13}$.

\section{ACKNOWLEDGEMENTS}

The research work was supported by National Natural Science Foundation of China under Grant No. 61273022.

\section{REFERENCES}

[1] Zhi-bin Li,Xin-xin Zhao,Wei Li. The inverse eigenvalue problem for generalized Jacobi matrices [J]. Journal of Dalian Jiaotong University,2008,29(4):6 10

[2] Yin Qing-xiang. Inverse eigenvalue problem for symmetric three-diagonal matrix and periodic three-diagonal matrix[J]. Computational physics,1995,12(4):467 474.

[3] Huang xian-tong. Inverse eigenvalue problem for symmetric three-diagonal matrix[J]. South metallurgical institute journa,1998,19(1):50 56. 